\title{
Proton and anti-proton distributions at RHIC
}

\author{
F.Videbæk ${ }^{1}$ \\ for The BRAHMS Collaboration \\ ${ }^{1}$ Physics Department, \\ Brookhaven National Laboratory, \\ NY 11973, USA
}

\begin{abstract}
Properties of transverse momentum spectra and rapidity dependence of protons and anti-protons in $\mathrm{Au}-\mathrm{Au}$ collisions at $\sqrt{s_{N N}}=200 \mathrm{GeV}$ are discussed. The net-proton yields are approximately constant at $|y|<1$ and increases towards $y \approx 3$. The mean rapidity loss is estimated to be in the range of $1.9<\delta y<2.4$.
\end{abstract}

Keywords: Heavy Ion Collisions, Nuclear Stopping, Baryon Distributions PACS: 25.75DW5

\section{Introduction}

The major goal for relativistic heavy ion reactions is to form hot and dense nuclear matter, to study its properties, and to come to a better understanding of nonperturbative QCD. The heavy ion experiments at RHIC have this as their main purpose. It is important to understand nuclear stopping in detail since it is a requisite for the formation of such systems in describing the conversion of the initial kinetic energy into matter excitation at mid-rapidity. A description in detail of how this transport takes place is a necessary ingredient in our overall understanding of the reactions and in the expectations to form and study the properties of QGP.

The early picture by Bjorken [1] proposes a transparent scenario in which the central region is net-baryon free, and all baryons are at high rapidity after the collision; as a second consequence the produced particles forms a rapidity plateau that exhibits boost invariance, i.e., constant yield and properties vs. rapidity. At the lower energies, at AGS and SPS the collisions are closer to a Landau description where most of the baryons end up near mid-rapidity $[2,3]$.

The exploration of the rapidity dependence of particle production and of stopping is one of the main motivations for the BRAHMS experiment at RHIC. In this contribution, preliminary data on proton and anti-proton distribution measured in 


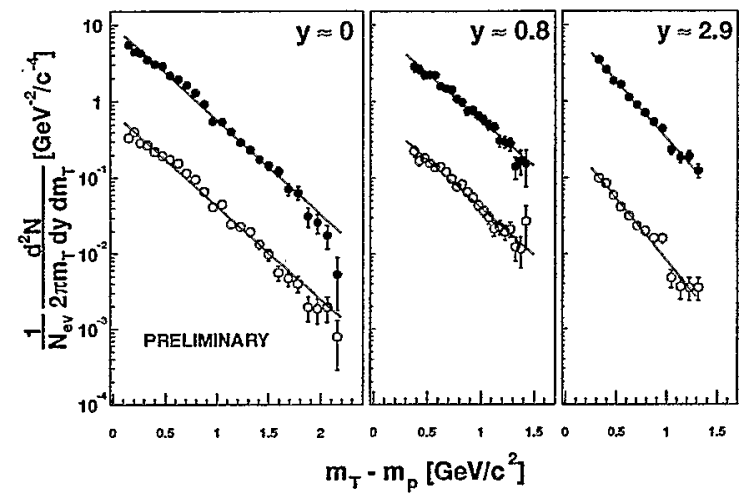

Fig. 1. Proton and anti-proton $\mathrm{m}_{t}$ spectra at 3 rapidities. The protons and anti-protons are indicated by filled and open circles, respectively. For clarity the $\overline{\mathrm{p}}$-spectra are divided by 10 . The errors are statistical only.

$\mathrm{Au}-\mathrm{Au}$ collisions at $\sqrt{s_{N N}}=200 \mathrm{GeV}$ are discussed.

\section{Experimental Setup}

The BRAHMS experiment measures identified, charged hadrons over a broad range of rapidity and transverse momenta with two moveable spectrometers [4]. The Mid-Rapidity Spectrometer (MRS) measures from $30^{\circ}<\Theta<90^{\circ}$ with particle identification from a Time-Of-Flight system with a time resolution of $\sigma \approx 75$ psec allowing for $\mathrm{p}-\mathrm{K}$ separation up to $3.5 \mathrm{GeV} / \mathrm{c}$. The Forward Spectrometer (FS) took data in $3^{\circ}<\Theta<20^{\circ}$ for 2001 RHIC run. In FS the Ring Imaging Cherenkov detector was used for particle identification of protons by vetoing of pions and kaons at momementa $\leq 17 \mathrm{GeV} / \mathrm{c}$ and by observing the predicted ring radius above. The momentum is provided from multiple tracking detectors consisting of time projections and drift chambers. For each data taking setting the geometrical acceptance was calculated using a Monte Carlo simulation of the BRAHMS detector. Corrections for multiple scattering, absorbtion, and annihilation have been calculated in the same framework. Tracking efficiencies have been estimated from simulations, and from the data using a subset of the detectors to define reference tracks. Typical overall tracking efficiencies for central collisions are $\approx 92 \%$ in MRS and $\approx 60 \%$ in FS. Centrality selection was done using the charged particle multiplicity in the mid-rapidity range of $-2<\eta<2$ as described in [5]. Most of the data presented here are from a centrality cut of of $0-10 \%$. 


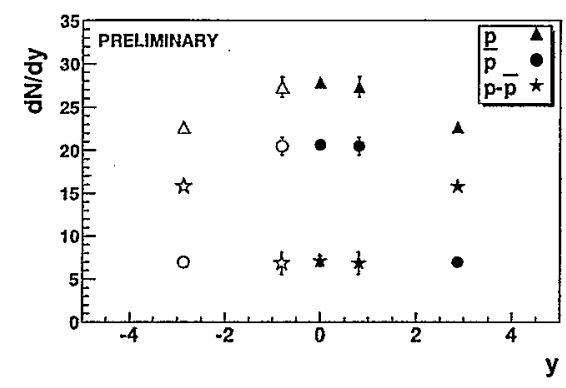

Fig. 2. The rapidity densities $\mathrm{dN} / \mathrm{dy}$ for $\mathrm{p}, \overline{\mathrm{p}}$ and $\mathrm{p}-\overline{\mathrm{p}}$ central collisions. Closed symbol are for measured points, and open are values obtained by reflecting the measured data around mid-rapidity.

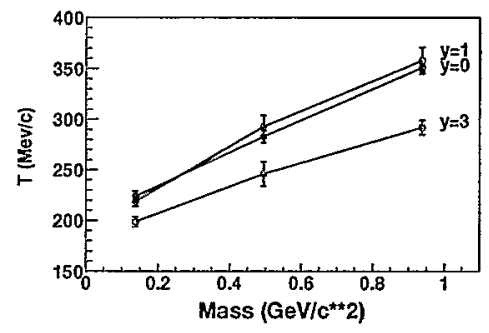

Fig. 3. Inverse slope parameters obtained from $m_{t}$-fits to $\pi^{+}, \mathrm{K}^{+}$, and proton spectra. The lines connects points from the same rapidity.

\section{Discussion}

The $p$ and $\bar{p}$ spectra are shown in Fig. 1 for the most $0-10 \%$ central events. The spectra are fitted with an $m_{t}$ exponential function to extract the inverse slopes parameters and yields. The measured yields are $\approx 80 \%$ of the extrapolated $\mathrm{dN} / \mathrm{dy}$ yields at $\mathrm{y}=0, \approx 60 \%$ at $\mathrm{y}=1$ and $\approx 40 \%$ at 3.5 Different parametric dependencies were employed at the higher rapidities, and the major systematic errors comes from the assumed extrapolation to low $m_{t}$. Since near mid-rapidity either a simple exponential $m_{t}$ description or Boltzman is appropriate, this was then assumed to be valid at the higher rapidities; still in the measured $p_{t}$ range we cannot distinguish between an exponential $m_{t}$ or $p_{t}$ description. At mid-rapidity the yield of $\bar{p}$ is $80 \%$ of protons and drop at the highest rapidity as shown by the rapidity densities in Fig. 2. Also displayed is the $N(\mathrm{p})-N(\overline{\mathrm{p}})$ yields. The ratio of $\overline{\mathrm{p}}$ to $\mathrm{p}$ vs. rapidity is discussed in more detail in [6]. The shape of the $p$ and $\bar{p}$ spectra are nearly identical. The inverse slopes for pions, kaons and protons are displayed in Fig. 3 for 3 different rapidity bins. It is observed that the inverse slopes drops at the higher rapidities, indicating a softening of the spectrum. At the same time a significant increase in inverse slope with mass is still seen at rapidity 3 consistent with the presence of transverse flow over all rapidities. More details are given in Ref. $[7,8]$.

The $N(\mathrm{p})-N(\overline{\mathrm{p}})$ distributions, though still preliminary and with additional data yet to come, give us important clues and information about the development of stopping with the collision energy. A summary of the $N(\mathrm{p})-N(\overline{\mathrm{p}})$ distributions is given in Fig. 4 showing selected data from AGS [9, 10], from NA49 at SPS [11], and the preliminary BRAHMS data [7]. A clear evolution is observed with near complete stopping at AGS, the development of a small dip near mid-rapidity, while at RHIC finally a low $N(\mathrm{p})-N(\overline{\mathrm{p}})$ is observed at mid-rapidity with the bulk of the protons to be found even outside the rapidity range measured so far. It is instructive to display the information from these data in two other ways. In the 


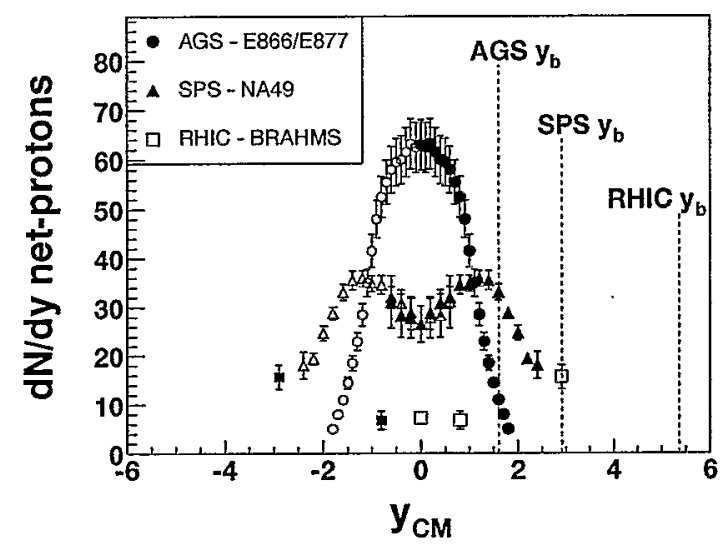

Fig. 4. Compilation of dN/dy from AGS to RHIC.

first, the $N(\mathrm{p})-N(\overline{\mathrm{p}})$ at mid-rapidity is displayed vs $\sqrt{s_{N N}}$ in Fig. 5. This figure, as well as those following, includes additional data from low energy $[10,12]$, and from the RHIC data $[13,14]$. Over the now available energy range in Heavy Ion collisions a drop of about an order of magnitude in net-proton number is observed.

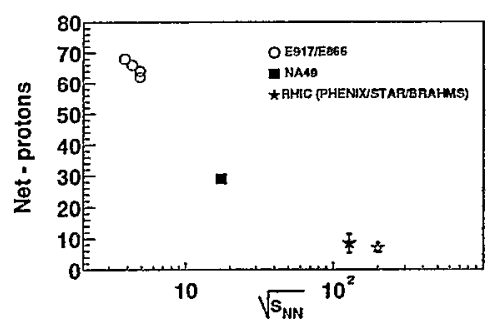

Fig. 5. $\sqrt{s_{N N}}$ dependence of Net-proton yield in AA collisions.

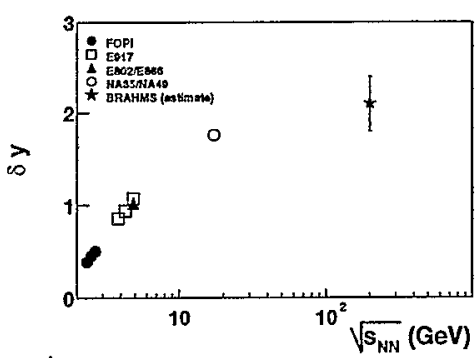

Fig. 6. $\sqrt{s_{N N}}$ dependence of $\delta y$ in AA collisions.

Even though we do not have measurements of the net-protons yields in the range from 3 to beam rapidity $\left(y_{b}\right)$ of 5.4 , we can attempt to estimate to mean rapidity loss, as done in Ref.[2], as $\delta y=\int_{0}^{y_{b}} d y\left(y_{b}-y\right) \frac{d N}{d y} / \int_{0}^{y_{b}} d y \frac{d N}{d y}$. The expected number of net-protons in the rapidity range 0 to 5.4 for the most central collisions with $\mathrm{N}_{\text {part }} \sim 340$ is 68 if the initial proton to neutron ratio is conserved or 85 if proton to neutron equilibration is assumed for all rapidities. The observed number is $\sim 31$. The non-observed net-protons have to be in the interval above rapitidty at one extreme with all remaining protons at rapidity 3.1 , an the other at beam-rapidity. 
This leads to an estimate of a $\delta y$ in the range of 1.7 to 2.4. Not all net-protons coming from hyperon decays are included in the experimental distributions. The effect on the $\delta y$ range estimate is small. The data from the lower energies and this the present estimate is plotted in Fig. 6 . There is a clear trend of an increase with energy, with values in central AA collisions a factor of two larger than in elementary pp collisions ( $\delta y \sim 0.7-0.9)$.

Comparisons of the measured net-proton yields with predictions from theoretical models are shown in Fig. 7. Models tested here are the ones using initial conditions based on PQCD and string dynamics which have been extensively compared with other measurements at RHIC: HIJING [15], HIJING/B [16], and AMPT [17]. The HIJING/B model with a baryon-junction mechanism implementedh leads to more baryon stopping than HIJING. A MultiPhase Transport Model, AMPT, also takes the initial conditions from HIJING, but includes re-scattering processes with some of the model parameters tuned to the heavy-ion data from SPS. The measurement and the models are in reasonable agreement within systematic errors at the near mid-rapidity, while HIJING somewhat underestimates the stopping at the forward region. The AMPT model is also able to account for the general trend of the rapidity-dependent $N(\bar{p}) / N(p)$ ratios [6] and the multiplicity distributions of charged particles [5] for the central collisions. It suggests that re-scattering plays a significant role in the dynamics of hadron distributions at RHIC energy. The microscopic transport model UrQMD [18] (not shown in Fig. 7), which reproduces many observed features at SPS energies predicts much higher stopping $\left(d N /\left.d y\right|_{p-\bar{p} \sim 12.5)}\right.$ at $y=0$.

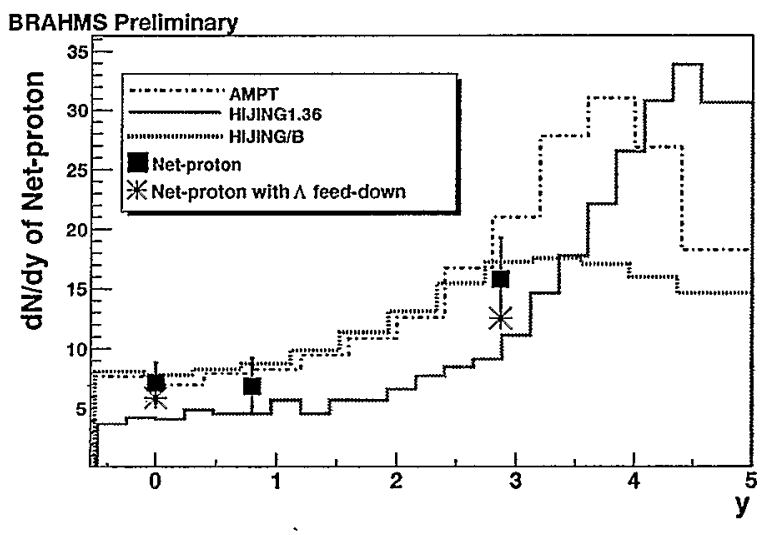

Fig. 7. Net-proton distributions compared with theoretical models described in text. The errors include both statistical and systematic uncertainty. Net-proton yields with $\Lambda$ feed-down corrections are shown at $y=0$ and 2.9 . 
F.Videbæk

\section{Summary}

BRAHMS has measured proton and anti-proton spectra at mid and forward rapidities in central $\mathrm{Au}+\mathrm{Au}$ collisions at $\sqrt{s_{N N}}=200 \mathrm{GeV}$. The net-proton yields indicate that at RHIC we observe a mid-rapidity region with a low net-baryon density $N(\mathrm{p})-N(\overline{\mathrm{p}}) \sim 7$ in a narrow rapidity range of $|y| \leq 1$. The $N(\mathrm{p})-N(\overline{\mathrm{p}})$ at mid-rapidity decreases significantly with $\sqrt{s_{N N}}$ dropping by a factor of 5 from SPS to RHIC energies. Still the net yield at mid-rapidity is higher than indicated by fully transparent models. The mean rapidity loss was estimated from the rapidity distribution under a set of different assumptions to be in the range of $1.7 \leq \delta y \leq 2.4$, i.e., a continued growth with energy and a factor of two larger than in elementary $\mathrm{pp}$ collisions.

\section{Acknowledgements}

This work was supported by the Division of Nuclear Physics of the Office of Science of the U.S. Department of Energy under contract with BNL (No.DE-AC0298CH10886).

\section{References}

1. J. D. Bjorken, Phys.Rev D27 (1983) 140

2. F. Videbæk and O. Hansen, Phys. Rev. C52 (1995) 2584.

3. F. Videbæk, proceeing of $17^{\prime}$ th Winter Workshop on Nuclear Dynamics.

4. M.Adamczyk et.al. BRAHMS Collaboration Nucl.Instr.Meth 499 (2003) 437.

5. The BRAHMS Collaboration Phys. Rev. Lett. 88 (2002) 202301

6. The BRAHMS Collaboration Phys. Rev. Lett. 90 (2003) 102301

7. J.H.Lee et al, BRAHMS Collaboration, Proceedings of QM02 Nucl.Phys A715 (2003)

8. D.Ouerdane et al, BRAHMS Collaboration, Proceedings of QM02 Nucl.Phys A715 (2003)

9. L. Ahle et al., E866 collaboration . Phys.ReV. C57 (1998) R466.

10. N. Herrmann, J. P. Wessels and T. Wienold, Ann. Rev. Nucl. Part. Sci. 49 (1999) 581.

11. H.Appelshäuser, The NA49 collaboration Phys.Rev.Lett. 82 (1999) 2471.

12. B. Back et al., E917 Collaboration. Phys. Rev. Lett. 86 (2001) 1970

13. C. Adler et al., STAR collaboration, Phys. Rev. Lett. 87 (2001) 262302;

Erratum Phys. Rev. Lett. (2003)

14. K. Adcox et al., Phys Rev Lett . 88(2002) 242301.

15. X.N. Wang and M. Gyulassy, Phys. Rev. D44 (1991) 3501

16. S.E. Vance, M. Gyulassy, and X.N. Wang, Nucl.Phys. A638 (1998) 395c

17. B. Zhang et al., Phys. Rev. C61 (2000) 067901

18. M.J. Bleicher et al, Phys. Rev. C62 (2000) 24904 